\title{
Interfacing polymers and tissues: Quantitative local assessment of the Foreign Body Reaction of Mononuclear Phagocytes to Polymeric Materials

\author{
--Manuscript Draft--
}

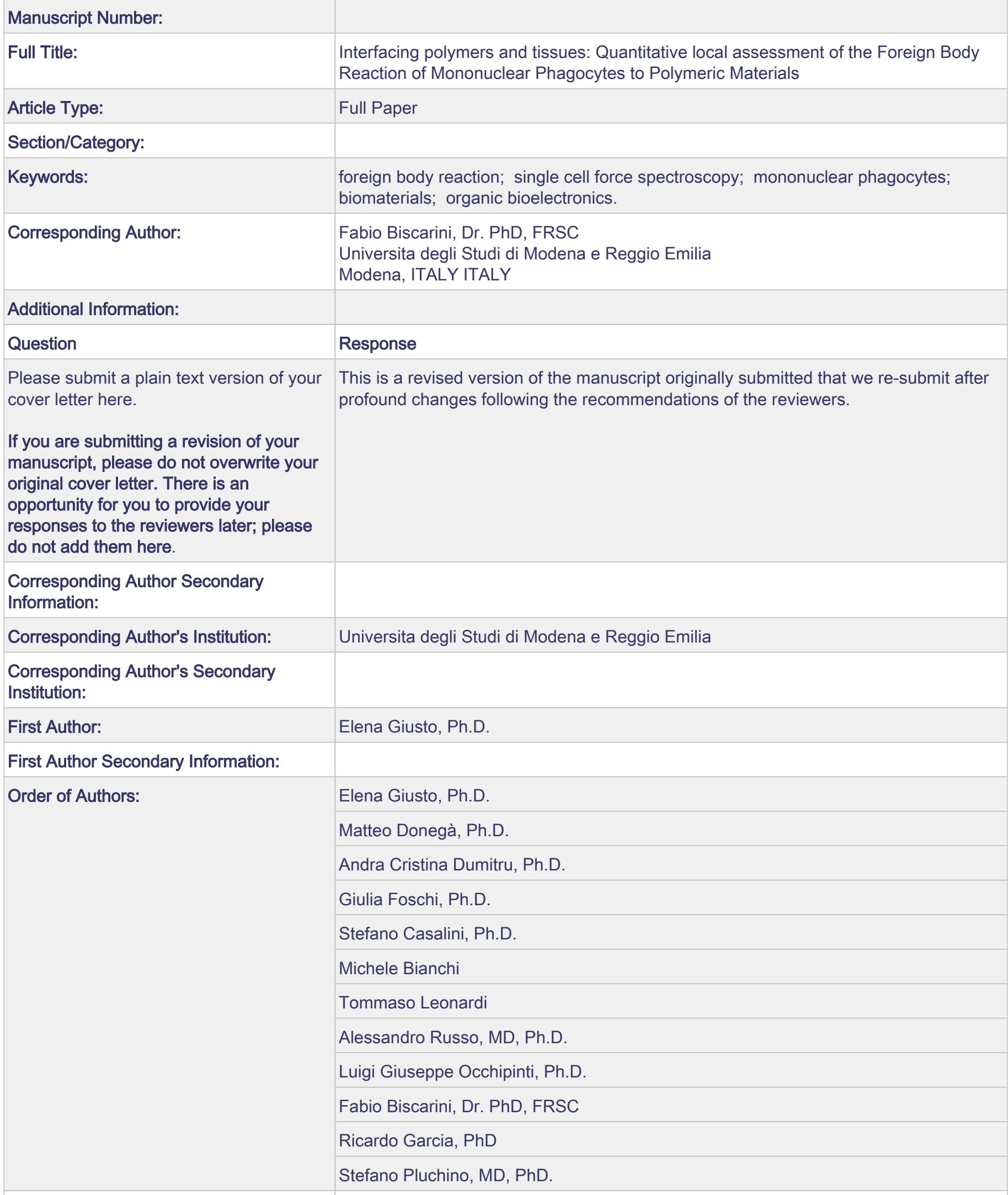

Order of Authors Secondary Information: 


\begin{tabular}{|l|l|}
\hline Abstract: & $\begin{array}{l}\text { A quantitative method to assess the in vitro FBR of MPs to polymers relevant in } \\
\text { implants for prosthetics, advanced therapies, and regenerative medicine is presented. } \\
\text { Our approach integrates single-cell force spectroscopy (SCFS) with immunogenic } \\
\text { profiles of the MPs. In the cell force spectroscopy experiments a single phagocyte, } \\
\text { linked at the end of an AFM cantilever, probes the adhesion forces between the cell } \\
\text { and the polymer surface. SCFS measures adhesion forces in a range from } 10 \text { pN to } \\
100 \mathrm{nN} \text { and with spatial resolution from the cell size down to nanometers, and } \\
\text { accesses the early adhesion events established at contact times between milliseconds } \\
\text { and minutes. We study the time evolution (1-60 s) of the adhesion force between the } \\
\text { phagocyte and the polymer surface before and after the treatment with an } \\
\text { immunosuppressive drug. Specifically, we use Minocycline, a Federal Drug } \\
\text { Administration (FDA)-approved third generation tetracycline with anti-inflammatory } \\
\text { effects. We find that the adhesion force values measured at the single cell level } \\
\text { correlate to the immunogenic profiles obtained by analysis of biomarkers and } \\
\text { morphology of the MPs in culture. }\end{array}$ \\
\end{tabular}


DOI: $10.1002 /(($ please add manuscript number $))$

\section{Interfacing polymers and tissues: Quantitative local assessment of the Foreign Body}

\section{Reaction of Mononuclear Phagocytes to Polymeric Materials}

Elena Giusto ${ }^{a}$, Matteo Donegà ${ }^{a}$, Andra C. Dumitru ${ }^{b}$, Giulia Foschic, $^{c,}$, Stefano Casalini ${ }^{c}$, Michele Bianchi $^{d}$, Tommaso Leonardi ${ }^{a, e}$, Alessandro Russo ${ }^{d}$, Luigi G. Occhipinti ${ }^{\dagger, \$}$, Fabio Biscarini $^{c *}$, Ricardo Garcia ${ }^{b}$ * and Stefano Pluchino ${ }^{a, *}$

aDepartment of Clinical Neurosciences, Wellcome Trust-Medical Research Council Stem Cell Institute and National Institute for Health Research Biomedical Research Centre, University of Cambridge, Hills Road, CB2 OHA Cambridge, UK;

bInstituto de Ciencia de Materiales de Madrid, CSIC, Sor Juana Inés de la Cruz 3, 28049 Madrid, Spain; 'Dipartimento di Scienze della Vita, Università di Modena and Reggio Emilia, Via Campi 103, 41125 Modena, Italy;

${ }^{d}$ Laboratorio di NanoBiotecnologie-Istituto Ortopedico Rizzoli, Via di Barbiano 1/10, 40136 Bologna, Italy; eThe EMBL-European Bioinformatics Institute, Wellcome Trust Genome Campus, Hinxton, Cambridge CB10 1SD, UK;

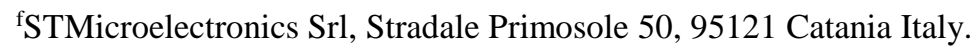

+Present address: Scriba Nanotecnologie S.r.1., Via di Corticella 183/8, 40128 Bologna, Italy

${ }^{\$}$ Present address: Department of Engineering, Electrical Engineering Division, University of Cambridge, 9 JJ Thomson Avenue, CB3 0FA Cambridge, UK.

\section{*Address correspondence to:}

Dr. Stefano Pluchino, MD, PhD, Department of Clinical Neurosciences, Wellcome TrustMedical Research Council Stem Cell Institute and National Institute for Health Research Biomedical Research Centre, University of Cambridge, Hills Road, CB2 OHA Cambridge, UK; Email: spp24@cam.ac.uk

Prof. Ricardo Garcia, PhD, Instituto de Ciencia de Materiales de Madrid, CSIC, Sor Juana Inés de la Cruz 3, 28049 Madrid, Spain; Email: r.garcia@csic.es

Prof. Fabio Biscarini, PhD, Dipartimento di Scienze della Vita, Università di Modena e Reggio Emilia, Via Campi 103, 41125 Modena, Italy; email: fabio.biscarini@unimore.it

Keywords: foreign body reaction, single cell force spectroscopy, mononuclear phagocytes, biomaterials, organic electronics, implants, Minocycline. 


\section{WILEY-VCH}

Synthetic biomaterials are widely used to fabricate medical devices regulating, replacing or restoring impaired functions of the body. These applications are expected to grow with the advent of advanced therapies based on loco-regional treatments or stem-cell grafts for tissue regeneration. ${ }^{12}$

The implantation of any material or medical device into a host tissue is likely to trigger an adverse foreign body reaction (FBR), ${ }^{3}$ which is a cascade of events strongly intertwined with the interactions between cells and materials. FBR starts with recruitment, adhesion and accumulation of white blood cells (leukocytes) - including neutrophils and monocytes/macrophages - at the tissue-implant interface. ${ }^{2}$ Upon adhesion, activated mononuclear phagocytes (MPs) fuse into giant cells in the attempt of engulfing the foreign body. Activated MPs release a plethora of pro-inflammatory signals such as cytokines, chemokines, reactive oxygen intermediates, nitric oxide and degrading enzymes to finally recruit additional immune cells and fibroblasts. ${ }^{2}$ In later chronic phases, fibroblasts and mast cells produce a collagen-based extracellular matrix that wraps the implanted material/device into a fibrotic, avascular capsule, thus preventing any interaction with the surrounding tissue and impairing the long-term functionality of the implant. ${ }^{4}$

The origin of FBR is not completely understood. Correlations have been assessed between bulk and surface materials properties, such as elasticity and plasticity, surface morphology and texture, wettability and surface tension, and the initial adhesion of cells to substrates. 678 $9{ }^{10}$ Because cell/material adhesion is a multiscale phenomenon, there is a lag in accessing the cell/material interaction from the single cell down to the molecular level.

Most of the studies focusing on FBR are based on phenomenological or semi-quantitative cell adhesion assays in vitro ${ }^{10}$. The established methodologies foresee the detection of relevant biomarkers expressed by cells of the immune system, together with the evaluation of cell morphological parameters. In vitro assays imply a lengthy response, large variances of the observables, and difficult standardization of the protocols. Advancing the methodology from the heuristic level to a robust quantitative characterization in a shorter timescale is important both for materials screening, as well as to evaluate the synergic effects of drugs and chemotrophic factors that may prevent or delay the onset of cell adhesion.

Here, we present a quantitative method to assess the in vitro FBR of MPs to polymers relevant in implants for prosthetics, advanced therapies, and regenerative medicine. Our approach integrates single-cell force spectroscopy (SCFS) with immunogenic profiles of the MPs. In the cell force spectroscopy experiments a single phagocyte, linked at the end of an AFM cantilever, probes the adhesion forces between the cell and the polymer surface. SCFS 


\section{WILEY-VCH}

measures adhesion forces in a range from $10 \mathrm{pN}$ to $100 \mathrm{nN}$ and with spatial resolution from the cell size down to nanometers, ${ }^{11}$ and accesses the early adhesion events established at contact times between milliseconds and minutes. ${ }^{12} 1314$ We study the time evolution (1-60 s) of the adhesion force between the phagocyte and the polymer surface before and after the treatment with an immunosuppressive drug. Specifically, we use Minocycline, a Federal Drug Administration (FDA)-approved third generation tetracycline with anti-inflammatory effects. We find that the adhesion force values measured at the single cell level correlate to the immunogenic profiles obtained by analysis of biomarkers and morphology of the MPs in culture, and that Minocycline reduces both pro-inflammatory gene expression profile as well as cell adhesion

We choose poly-dimethylsiloxane (PDMS) - a biocompatible non-biodegradable polymer and poly-(lactic-co-glycolic acid) (PLGA) - a biocompatible and biodegradable polymer - as prototypical polymeric materials for biomedical applications. ${ }^{15} 16$ They are cast as films with $100 \mu \mathrm{m}(\mathrm{PDMS})$ and $5 \mu \mathrm{m}$ (PLGA) thickness. Cells are usually seeded on polystyrene (PS) petri-dishes, hence this material is used as the control interface for mechanical and topographical properties. In addition, Lypopolysaccharide (LPS) is used as positive proinflammatory stimulator. Our specific interest on PDMS and PLGA arises from their potential use as scaffolds for organic electronic implants, whose operation time scale is tailored to a few months, for the advanced treatment of pathologies of the nervous system as for instance spinal cord injury.

The morphological parameters and mechanical properties measured on the PS, PLGA and PDMS surfaces are reported in Table 1. All surfaces exhibit a smooth featureless morphology with a (saturated) root-mean-square (rms) roughness $<5 \mathrm{~nm}$ (AFM images not shown). The mechanical properties of PS, PLGA, PDMS are measured by nanoindentation. The reduced elastic modulus $E_{r}$ is below $100 \mathrm{MPa}$ for PDMS, more than 10 times softer than PS (5 GPa) and PLGA (6.5 GPa). PDMS exhibits a low contact hardness $H_{c}$ value (5 MPa) compared to PLGA (150 MPa) and PS (about $250 \mathrm{MPa}$ ).

To evaluate the medium-term interaction of MPs with substrates, we quantify the number and morphology of adhering cells from fluorescence images. MPs exhibit different morphologies: small and rounded when plated on PDMS, large and spread when plated on PLGA and PS (with or without addition of LPS) (Figure 1a). Significant differences are observed in the number of cells adhering to the materials, with PDMS showing the lowest and 


\section{WILEY-VCH}

PLGA the highest numbers of adhering MPs both after 1 hour (data not shown) and 24 hours in vitro (Figure 1b), respectively. As the fraction of proliferating cells was only about 1-2\% (over total plated) on all of the materials tested (data not shown), we confirm that the above difference depends on the different adhesive response of MPs, and not on the proliferation rates, consistently with previous observations on organic thin films. ${ }^{19}$

The MPs morphology is quantified by extracting the average projected area of the cells (Figure 1c) and their elongation factor (Figure 1d), a parameter that has been previously associated with the functional state of MPs. ${ }^{20}$ Cell adhesion and migration are also accompanied by the formation of filopodia, thin spike-like cytoplasmic outgrowth containing bundles of parallel actin filaments that act as fingers probing the microenvironment in adhering/migrating cells. ${ }^{21}$ PLGA promotes extensive formation of filopodia, while PDMS shows an opposite effect leading to smooth cell contours (Figure 1e).

We then assess the activation of MPs by profiling the expression levels of the proinflammatory genes Tumor necrosis factor- $\alpha(\operatorname{Tnf}-\alpha)$, Interleukin $1 \beta(I l 1 b)$, Il6 and Nitric oxide synthase (Nos2) as well as of the anti-inflammatory genes Arginase 1 (Argl) and Mannose Receptor I (MrcI) at 6 and 24 hours after plating in vitro. These are classical markers for pro-inflammatory (M1) and anti-inflammatory (M2) macrophages. ${ }^{21,22}$ The results are shown in Figure 2 a-f. PLGA shows a prolonged pro-inflammatory activation of MPs lasting until 24 hours, whereas PDMS shows a similar profile at 6 hours, which is then reduced at 24 hours leading to the lowest pro-inflammatory response of MPs, when compared to PS.

Correlation analysis (Figure 2g) between materials properties and their FBR profile reveales a direct correlation between MPs adhesion and material capacitance, elastic modulus, hardness and porosity. We also observe a direct correlation between the induction of proinflammatory genes in MPs (Illb, Il6 and Nos2) and the material hardness and elastic modulus. Elastic work, roughness, contact angle and porosity show direct correlation with the expression levels of the pro-inflammatory cytokine Tnf. These data confirm the opposite effects induced by PLGA (high) and PDMS (low) on MPs.

To elucidate the interactions occurring between MPs and polymer films we perform SCFS measurements in a serum-free setting. In our approach the cantilever is amino-functionalized and coated with fetal bovine serum. Then a single MP is linked to the end of the cantilever, 


\section{WILEY-VCH}

with the aim of measuring the force as a function of the probe-surface distance (force curve). The analysis of force curves recorded while detaching a single MP from the substrate suggests a complex interaction characterized by (1) a sharp increase of the adhesion force corresponding to the elastic stretching of the entire cell; (2) a sequence of de-adhesion steps attributed to the unbinding of cell membrane proteins from the surface; (3) a region of plateaus of constant force extending around $1000 \mathrm{~nm}$ distance due to the detachment from the surface of micrometer-long membrane tethers; (4) a complete detachment of the cell from the surface (Figures 3a-b).

To compare the strength of adhesion on PLGA (Figure 3a) and PDMS (Figure 3b), we measure the adhesion forces of MPs for contact times of 10 and $30 \mathrm{~s}$ (Figure 3c). The data include 20 cells and each measurement involves the acquisition and average of 10 force curves per each contact time. We measure >1.5-fold higher adhesion forces on PLGA (492 \pm $118 \mathrm{pN}$ at $10 \mathrm{~s}$ and $805 \pm 269 \mathrm{pN}$ at $30 \mathrm{~s})$ than PDMS $(306 \pm 73 . \mathrm{pN}$ at $10 \mathrm{~s}$ and $409 \pm 121 \mathrm{pN}$ at 30s), which increases upon longer contact time only on PLGA. We also observe a larger standard deviation on PLGA than on PDMS. The variation coefficients (ratio of standard deviation to mean) on PDMS and PLGA are comparable (0.23 at $10 \mathrm{~s}, 0.3$ at $30 \mathrm{~s})$. This indicates that the force curves measured between MPs and PDMS result from similar interactions and the greater mean and dispersion in the case of PLGA arise from an increasing number of the adhesion proteins in the extracellular membrane expressed by the MP interacting with PLGA.

Based on the above observations, and the relevance of PLGA for the fabrication of bioresorbable implants, we test the effects of Minocycline on the adhesion and activation of MPs on PLGA. MPs are plated on PLGA and treated for $24 \mathrm{~h}$ with two different doses $(10 \mu \mathrm{M}$ and $50 \mu \mathrm{M}$ ) of Minocycline. As shown in Figure 4a-b, $50 \mu \mathrm{M}$ Minocycline is effective in reducing the formation of filopodia of MPs seeded on PLGA. Minocycline-treated MPs are also analyzed by means of SCFS at increasing contact times of 5, 10, 30 and 60 s on PLGA. The data set includes $n \geq 15$ cells and $n \geq 3$ force curves per contact time. Figure $4 \mathbf{c}$ shows the adhesion force as a function of the contact time for untreated and Minocycline-treated cells. The mean value of the adhesion force at $5 \mathrm{~s}$ seems not to be influenced by the Minocycline treatment. However, significant differences are recorded for longer contact times. The adhesion force of untreated cells shows a three-fold increase with time $(527 \pm 174 \mathrm{pN}$ at $5 \mathrm{~s}$ and $1469 \pm 502 \mathrm{pN}$ at $60 \mathrm{~s}$ ), while the mean value of Minocycline-treated cells remains almost 


\section{WILEY-VCH}

constant in the $5-30 \mathrm{~s}$ range $(486 \pm 101 \mathrm{pN}$ at $5 \mathrm{~s}$ and $578 \pm 204$ at $30 \mathrm{~s})$ with an increase at $60 \mathrm{~s}$ $(754 \pm 360 \mathrm{pN})$ which is not statistically significant.

These results suggest that Minocycline effectively decreases the adhesion force between MPs and PLGA. Importantly, Minocycline does not affect MPs viability at any concentration tested (data not shown). Minocycline (both 10 and $50 \mu \mathrm{M}$ ) is also able to reduce MP activation, as shown by the down-regulation of Tnf, Illb, Il6 and Nos 2 at any concentration tested (Figure 5. The finer mechanism behind the action of Minocycline is not accessible to our comparative experiment, and further studies are required. The outcome is that, albeit PLGA is clearly immunogenic, the FBR against PLGA can be reduced by the local supply of Minocycline.

In conclusion, we demonstrate the existence of a correlation between the adhesion force of MPs to synthetic polymer surfaces and their activation towards FBR in vitro. In particular, the large mean value and spread of the adhesion force is related to the pro-inflammatory response of the MPs. The action of an anti-inflammatory drug such as Minocycline on the MPs yields a decrease of their adhesive force, and hence inhibits the FBR induced by PLGA. Our evidence hints to the molecular basis of FBR, and clearly indicates that adhesion is a potential target to minimize the FBR against materials that are immunogenic a priori.

SCFS provides a much faster (on the seconds to minutes time scale) indication than the morphological analysis and biomarkers profile (on the 24 hour time scale) on the affinity between cells of the immune system and the materials surface. Cell adhesion strength can be quantified by means of SCFS with high sensitivity and the adhesion dynamics monitored in real time. In SCFS there is no need for fluorescent labelling of the samples, or to fix the cells, and, furthermore, the data analysis is not as time consuming and operator-dependent as in the case of the simple morphological analysis. SCFS is therefore an effective tool to screen the immunogenic potential of materials and assess in vitro the efficacy of chemical or pharmacological treatments.

\section{Experimental Section}

\section{Preparation of materials}

PLGA (molecular weight: 66000 - 107000) composed by a 75:25 ratio of D,L-lactide and glycolide units was purchased from Sigma-Aldrich (P1941) and used as received. PLGA films were prepared by solution casting method. PLGA is dissolved in dichloromethane upon stirring for about 40 minutes at room temperature, to obtain a $1 \%$ wt solution. A volume of $100 \mu \mathrm{L}$ of this solution is then cast onto a cleaned glass slide (Thermo Scientific) into a square frame (Sigma, S1815, Secure Slip $^{\mathrm{TM}}$ glass coverslip silicone $1.2 \mathrm{~cm} \times 1.2 \mathrm{~cm}$ ) to obtain a thin 


\section{WILEY-VCH}

layer of the solution with defined geometry and thickness. The solvent is allowed to evaporate at $55^{\circ} \mathrm{C}$ for 5 hours in an oven, and then the frame is removed. The resulting transparent film is disinfected in $99 \%$ ethanol for 15 minutes and dried in air before using it. We measured a film thickness of approximately $5 \mu \mathrm{m}$.

PDMS (Sylgard 184, Dow Corning) was mixed in a 10:1 ( $\mathrm{w} / \mathrm{w})$ ratio of silicone and curing agent and placed in a vacuum chamber. The PDMS films (100 $\mu \mathrm{m}$-thick) have been obtained by spin coating (spin speed $500 \mathrm{rpm}$, acceleration $500 \mathrm{rpm} / \mathrm{s}$ and duration time 3 minutes) and post-baking in an oven at $120^{\circ} \mathrm{C}$ for 1 hour.

Polystyrene tissue culture-treated 24-well plates (Corning, \#07-200-84) were used as controls.

\section{Amplitude modulation AFM imaging}

The topography of PLGA, PDMS and PS was measured in air environment with a Nanowizard III AFM (JPK Instruments, Germany) in amplitude modulation AFM by mechanically driving the cantilever ${ }^{24}$. Rectangular PPP-NCH (Nanosensors, Switzerland) cantilevers with a nominal force constant $k=40 \mathrm{~N} \mathrm{~m}^{-1}$ and a resonant frequency of $291 \mathrm{kHz}$ were employed in these measurements. Images were then processed with the JPK Data Processing Software and the roughness value was extracted (root-mean square, rms).

\section{Nanoindentation tests}

Mechanical tests were performed in air and at $21^{\circ} \mathrm{C}$ using a standard nanoindentation tester (NHT2, CSM Instruments, Peseux, Switzerland) equipped with a Berkovich diamond tip (plain strain modulus: $75.1 \pm 0.4 \mathrm{GPa}$, estimated silica Poisson's ratio: 0.16 ). The reduced elastic modulus $\left(\mathrm{E}_{\mathrm{r}}\right)$ and the contact hardness $\left(\mathrm{H}_{\mathrm{c}}\right)$ were estimated from the load $(\mathrm{P})$ vs. depth (h) curve according to the method by Oliver and Pharr. ${ }^{25} \mathrm{E}_{\mathrm{r}}$ was calculated from the slope of the unloading curve in the region between 40 and $98 \%$ of the maximum load according to:

$$
\mathrm{E}_{\mathrm{R}}=\frac{\sqrt{\pi}}{2} \frac{S}{\sqrt{A_{c}}},
$$

where $\mathrm{S}$ is the contact stiffness, calculated from the initial slope of the unloading curve $(\mathrm{dP} / \mathrm{dh})$, and $\mathrm{A}_{\mathrm{c}}$ is the contact area.

$\mathrm{H}_{\mathrm{c}}$ was calculated as:

$$
\mathrm{H}_{\mathrm{c}}=\frac{P_{\max }}{A_{c}},
$$

where $\mathrm{P}_{\max }$ is the maximum applied load. 


\section{WILEY-VCH}

A linear loading (loading and unloading rate set at $1.8 \mathrm{mN} / \mathrm{min}$ ) for a maximum load of 0.3 $\mathrm{mN}$ was used. Due to the extreme elastic behavior of PDMS, a linear loading (loading and unloading rate set at $120 \mathrm{mN} / \mathrm{min}$ ) for a maximum load of $20 \mathrm{mN}$ was required. The maximum loads were chosen in order to achieve a maximum indentation smaller than the $10 \%$ of film thickness, thus to strongly limit the contribution of the mechanical properties of the substrate. ${ }^{26}$ In order to limit the viscoelasticity contribution due to the time dependent behavior of investigated samples, a creep hold of 60 s has been introduced at peak load. ${ }^{27,28}$ The control of the thermal drift was automatically performed by the system between each indentation. All the data resulting from explicitly inadequate contact points were discarded from the data set analysis. At least eight indentation measurements were performed on different areas within each sample and the results provided as mean $\pm \mathrm{SD}^{28}$.

\section{Contact Angle Measurements}

The contact angle measurements were performed by a Digidrop GBX Model DS on droplets $(0.7 \mu 1)$ of bi-distilled water placed on the polymer substrates at RT.

\section{Single Cell Force Spectroscopy (SCFS)}

Cell adhesion measurements were conducted using a Nanowizard III AFM (JPK Instruments, Germany) mounted on top of an inverted optical microscope (Axiovert A1, Zeiss, Germany). To attach a single cell, the cantilever was lowered towards the petri dish at a speed of $5 \mu \mathrm{m} / \mathrm{s}$ and pressed onto a cell by applying a contact force of $3 \mathrm{nN}$ for $5 \mathrm{~s}$. Then the cantilever was pulled $50 \mu \mathrm{m}$ away from the sample surface. A resting period of 10 minutes was introduced before the adhesion measurements. The microcantielver with the cell attached to it was then approached towards the relevant material surface at a speed of $5 \mu \mathrm{m} \mathrm{s}^{-1}$ until reaching a contact force of $0.3-0.5 \mathrm{nN}$.

All reagents used for the cantilevers functionalization were purchased from Sigma Aldrich (Spain). Tipless microcantilevers (NPO-10, Bruker, USA) were first cleaned thoroughly by immersion in a solution of $0.25 \mathrm{M}$ sulphuric acid and $9.8 \mathrm{M}$ hydrogen peroxide $4: 1(\mathrm{v} / \mathrm{v})$ for 30 minutes. The cantilevers were rinsed with ultrapure water and then immersed into a solution of APTES-water-ethanol 5:5:90 (v/v/v) for 30 minutes. The resulting aminofunctionalized microcantilevers were rinsed with ultrapure water, ethanol, dried with nitrogen gas and stored in a dry atmosphere. The microcantilevers were finally immersed in $10 \%$ FBS at $4^{\circ} \mathrm{C}$ overnight, rinsed ten times in 0.01 M PBS ( $\left.\mathrm{pH} 7.4\right)$ and stored at $4{ }^{\circ} \mathrm{C}$ in a glass Petri dish. The tipless cantilevers (nominal spring constant $0.06 \mathrm{~N} / \mathrm{m}$ ) were calibrated before 


\section{WILEY-VCH}

starting the cell adhesion measurements. The optical lever sensitivity was calibrated by acquiring deflection $v s$. distance curves on a hard surface (mica). One hundred deflection- $v s$ distance curves were acquired and the sensitivity of the photodiode was calculated as the mean value of the slope of the deflection curve measured in the repulsive region. The force constant $k$ and quality factor of the cantilevers were determined by using the thermal noise method. ${ }^{29}$ The calibrated $k$ was found in the 0.05 to $0.07 \mathrm{~N} / \mathrm{m}$ range. The force was then calculated by using Hooke's law, $F=-k \cdot \Delta z$, where $\Delta z$ is the cantilever deflection.

\section{Isolation and differentiation of MPs from the bone marrow}

All the procedures were performed accordingly to the principles of laboratory animal care approved by the UK Home Office animals (scientific procedures) act 1986. Bone marrowderived monocytes were isolated from adult C57BL/6 male mice (Jax®)..$^{30}$

Briefly, mice were euthanized followed by neck dislocation. Femurs and tibias were collected, cleaned of the muscles and flushed using 25-gauge needles mounted on $10 \mathrm{ml}$ syringes filled with high glucose DMEM medium (Life Technologies, \#41966-029). A hypotonic solution ( $\mathrm{NH} 4 \mathrm{Cl} 0.8 \%, \mathrm{pH} 7.5)$ was used to lyse and remove red cells from the cell suspension. To obtain bone marrow-derived macrophages (MPs), monocytes were filtered through a $0.2 \mu \mathrm{m}$ cell strainer and plated with high glucose DMEM supplemented with $10 \%$ fetal bovine serum (FBS, Gibco Life Technologies, \#10500-064), Penicillin-Streptomycin (Life Technologies, \#15140-122) and 20\% L929 (Sigma-Aldrich, \#85011425) conditioned medium as a source of macrophage colony stimulating factor (mCSF) for 6 to 8 days at $37^{\circ} \mathrm{C}$, $5 \% \mathrm{CO}_{2}$. Upon differentiation, MPs were detached with cold phosphate buffer saline (PBS) and plated with fresh complete medium (with only $10 \%$ of L929 conditioned-medium) on PS or on different materials at a density of 27.000 cells $/ \mathrm{cm}^{2}$, unless differently specified, for the different time points needed.

\section{Minocycline preparation}

Minocycline hydrochloride (Sigma-Aldrich, \#M9511) was dissolved in DMEM at an initial concentration of $5 \mathrm{mM}$, sonicated, and sterilized through a $0.2 \mu \mathrm{m}$ filter. Minocycline solution was administered at final concentrations of 10 and $50 \mu \mathrm{M}$. Because of its short halflife, the same amount of Minocycline was added after 12 hours from the initial addition to the medium. 
Immunocytochemistry and Immunohistochemistry

MPs were fixed with pre-warmed 2\% PFA and 2\% sucrose in PBS for 5-10 min at RT and subsequently washed 3 times with PBS and conserved at $4{ }^{\circ} \mathrm{C}$ with $0.005 \%$ PBS sodium azide. Fixed cells were then incubated 30' at RT with 555 conjugated Phalloidin (Life Technologies, \#A34055) diluted 1:100 in blocking solution [PBS + 10\% Normal Goat Serum (NGS, PAA \#B11-035)]. MPs were washed three times in 1X PBS and incubated for 3 min with 4',6diamidino-2-phenylindole (DAPI, 1:10.000 in 1X PBS) at RT in the dark. Finally, cells were washed twice with PBS, once with distilled water and mounted on glass microscope slides with mounting medium (DAKO, \#S3023). Slides were stored at 4 or $-20^{\circ} \mathrm{C}$.

Propidium iodide (PI) and Annexin V assay kit has been used to test cell viability. Cells were harvested, centrifuged at $1300 \mathrm{rpm}$ for $3 \mathrm{~min}$ and the pellet carefully resuspended in 50 $\mu 1$ of staining buffer (10X Annexin V Binding buffer, eBioscience, \#00-0055-56 diluted 1:10 in distilled water) containing Annexin V and PI (both diluted 1:50) and incubated for $20 \mathrm{~min}$ at RT in the dark. Cells were then diluted with additional $150 \mu \mathrm{l}$ of buffer and analyzed with a flow cytometer.

\section{Quantitative gene expression analysis}

At the appropriate time point, MPs were collected from materials or PS using a cell scraper. For cells plated on materials (and their controls), total RNA was extracted using the miRCURYTM RNA Isolation Kit - Cell \& Plant (Exiqon, \#300110) accordingly to manufacturer instructions. Tetro cDNA Synthesis Kit (Bioline, \#BIO-65043) was used to obtain cDNA starting from $100 \mu \mathrm{g}$ of RNA. For bigger samples, cell pellets were resuspended with TRIzol® reagent (Life Technologies, \#15596-026) and total RNA was extracted following manufacturer description. 1000ng of RNA were converted into cDNA using high capacity cDNA reverse transcription kit (Applied Biosystem, \#4368813). For low quantity samples, RTq-PCR was performed starting from $10 \mathrm{ng}$ of cDNA using TaqMan® Fast Universal PCR Master Mix (Life Technologies, \#4352042) and read with a 7500 Fast Real Time PCR system machine (Applied Biosystem). The $\Delta \Delta \mathrm{Ct}$ method was used for quantification of gene expression. Expression levels were normalized to $\beta$-actin mRNA. All the experiments have been performed at least three times (three independent biological replicates).

Microscopy and image analysis 


\section{WILEY-VCH}

Fluorescent images were acquired using a Leica DMI 4000B inverted fluorescence microscope equipped with a Leica DFC3000 G camera or a Leica SPE DMI4000B scanning laser confocal microscope. For cell morphology analysis the long axis and short axis of each cell were manually traced and measured with Fiji software. The number of filopodia and the elongation factor were measured from 50 cells per experiment, selecting 5 isolated cells per field.

\section{Correlation analyses}

Heatmaps were generated in $\mathrm{R}$ using the function heatmap. 2 implemented in the gplots package. The expression data and the measurements of the materials properties were scaled by subtracting to each value the mean value across all materials and dividing by the standard deviation of the mean. The Pearson correlation coefficients between measurements were calculated using the cor() function in R. The row and column ordering as well as the dendrograms were obtained using the default hclust() function (complete method) on the distances computed by the dist() function (Euclidean distance).

\section{Statistical analysis}

Statistical analyses were performed using GraphPad Prism 5.0 software. One-Way analysis of variance (ANOVA) followed by Bonferroni's post hoc test correction was used for multiple group comparison, unless otherwise stated.

\section{Acknowledgements}

We thank Daniel Simon and Magnus Berggren from Linkoeping University, Sweden for discussing the experiments and providing us the PET samples. This research was supported by grants from: the EU 7th Framework Programme [FP7/2007-2013] under Grant Agreement No. 280772, "Implantable Organic Nanoelectronics (I-ONE-FP7)" project, the European Research Council (ERC) 2010-StG (RG 260511-SEM_SEM to SP); The Evelyn Trust (RG 69865 to SP), The Bascule Charitable Trust (RG 75149 to SP), The Great Britain Sakakawa Foundation to SP; MINECO (Spain) through project CSD2010-00024 and ERC-AdG-340177 to RG, and core support grant from the Wellcome Trust and MRC to the Wellcome Trust - Medical Research Council Cambridge Stem Cell Institute.

Author contributions. EG and MD contributed equally to this work. 


\section{WILEY-VCH}

EG, MD, TL and SP designed the MP-on-materials experiments; EG, MD and TL performed the experiments, acquired and analysed the data; EG, MD and SP interpreted the data. LGO designed and provided PI and PEN substrates. ACD and RG designed and analysed the SCFS experiments. ACD performed the SCFS and AFM measurements. GF, FB designed and made PLGA and PDMS films. GF, SC, MB, AR characterized the materials. FB integrated experimental results. $\mathrm{EG}, \mathrm{MD}, \mathrm{RG}, \mathrm{SP}, \mathrm{FB}$ wrote the manuscript.

Received: ((will be filled in by the editorial staff))

Revised: ((will be filled in by the editorial staff)) Published online: ((will be filled in by the editorial staff))

\section{References}

1. S. N. Jayasinghe, Biomatter, 2013, 3.

2. J. M. Anderson, A. Rodriguez and D. T. Chang, Semin. Immunol., 2008, 20, 86-100.

3. D. T. Luttikhuizen, M. C. Harmsen and M. J. Van Luyn, Tissue Eng., 2006, 12, 19551970 .

4. D. F. Williams, Biomaterials, 2008, 29, 2941-2953.

5. E. M. Hetrick, H. L. Prichard, B. Klitzman and M. H. Schoenfisch, Biomaterials, 2007, 28, 4571-4580.

6. P. Moshayedi, G. Ng, J. C. Kwok, G. S. Yeo, C. E. Bryant, J. W. Fawcett, K. Franze and J. Guck, Biomaterials, 2014, 35, 3919-3925.

7. T. O. Collier, J. M. Anderson, W. G. Brodbeck, T. Barber and K. E. Healy, J Biomed. Mater. Res. A, 2004, 69, 644-650.

8. R. J. Schutte, L. Xie, B. Klitzman and W. M. Reichert, Biomaterials, 2009, 30, 160168.

9. E. F. Irwin, K. Saha, M. Rosenbluth, L. J. Gamble, D. G. Castner and K. E. Healy, J. Biomat. Sci., 2008, 19, 1363-1382.

10. J. S. Hayes, E. M. Czekanska and R. G. Richards, Adv. Biochem. Eng. Biotechnol., 2012, 126, 1-31.

11. J. Helenius, C. P. Heisenberg, H. E. Gaub and D. J. Muller, J. Cell Sci., 2008, 121, 1785-1791. 
12. A. V. Taubenberger, D. W. Hutmacher and D. J. Muller, Tissue Eng. Part B Rev., 2014, 20, 40-55.

13. M. Benoit, D. Gabriel, G. Gerisch and H. E. Gaub, Nat Cell Biol., 2000, 2, 313-317.

14. E. A. Evans and D. A. Calderwood, Science, 2007, 316, 1148-1153.

15. I. R. Minev, P. Musienko, A. Hirsch, Q. Barraud, N. Wenger, E. M. Moraud, J. Gandar, M. Capogrosso, T. Milekovic, L. Asboth, R. F. Torres, N. Vachicouras, Q. Liu, N. Pavlova, S. Duis, A. Larmagnac, J. Voros, S. Micera, Z. Suo, G. Courtine and S. P. Lacour, Science, 2015, 347, 159-163.

16. A. Campana, T. Cramer, D. T. Simon, M. Berggren and F. Biscarini, Adv. Mater., 2014, 26, 3874-3878.

17. S. M. Krause, C.J.; Armstrong, R.D.; Ho, W.O. , J. Appl. Electrochem .,1997, 27, 291298.

18. C. A. McNeil, D.; Ball, M.; Ho, W., Anal. Chem., 1995, 67, 3928-3935.

19. I. Tonazzini, E. Bystrenova, B. Chelli, P. Greco, P. Stoliar, A. Calo, A. Lazar, F. Borgatti, P. D'Angelo, C. Martini and F. Biscarini, Biophys. J., 2010, 98, 2804-2812.

20. F. Y. McWhorter, T. Wang, P. Nguyen, T. Chung and W. F. Liu, Proc. Natl. Acad. Sci. U S A, 2013, 110, 17253-17258.

21. G. H. Ghassabeh, P. De Baetselier, L. Brys, W. Noël, J. A. Van Ginderachter, S. Meerschaut, A. Beschin, F. Brombacher, G. Raes, Blood. 2006, 108, 575-83.

22. K. A. Kigerl, J. C. Gensel, D. P. Ankeny, J. K. Alexander, D. J. Donnelly, P. G. Popovich, J Neurosci., 2009, 29,13435-44.

23. P. K. Mattila and P. Lappalainen, Nat. Rev. Mol. Cell Biol., 2008, 9, 446-454.

24. R. Garcia and A. San Paulo, Phys. Rev. B, 1999, 60, 4961-4967.

25. W. C. Oliver and G. M. Parr, J Mater Res 2004,19, 3-20.

26. R. Saha and W. D. Nix, Acta Mater., 2002, 50, 23-38.

27. T. Ishimoto, T. Nakano, M. Yamamoto, Y. Tabata, J. Mater. Sci. Mater. Med. 2011, $22,969-76$

28. M. Bianchi, M. Boi, M. Sartori, G. Giavaresi, N. Lopomo, M. Fini, A. Dediu, A. Tampieri, M. Marcacci and A. Russo, J. Mater. Sci: Mater. Med., 2015, 26, 1-9.

29. H. J. Butt and M. Jaschke, Nanotechnology, 1995, 6, 1.

30. V. A. Deshmukh, V. Tardif, C. A. Lyssiotis, C. C. Green, B. Kerman, H. J. Kim, K. Padmanabhan, J. G. Swoboda, I. Ahmad, T. Kondo, F. H. Gage, A. N. Theofilopoulos, B. R. Lawson, P. G. Schultz and L. L. Lairson, Nature, 2013, 502, 327-332. 


\section{WILEY-VCH}

Table 1. Morphological parameters and mechanical properties of the polymer substrates investigated.

\begin{tabular}{|l|c|c|c|}
\hline $\begin{array}{c}\text { Polymer } \\
\text { substrate }\end{array}$ & $\begin{array}{c}\text { rms Roughness } \\
(\mathbf{n m})\end{array}$ & $\begin{array}{c}\text { Reduced Young's } \\
\text { Modulus Er }(\mathbf{G P a})\end{array}$ & $\begin{array}{c}\text { Hardness Hc } \\
\mathbf{( M P a})\end{array}$ \\
\hline PDMS & $2.5 \pm 1.0$ & $0.10 \pm 0.02$ & $10 \pm 1$ \\
\hline PLGA & $0.5 \pm 0.2$ & $6.5 \pm 0.3$ & $175 \pm 20$ \\
\hline PS & $3.0 \pm 0.5$ & $5.5 \pm 0.5$ & $245 \pm 20$ \\
\hline
\end{tabular}
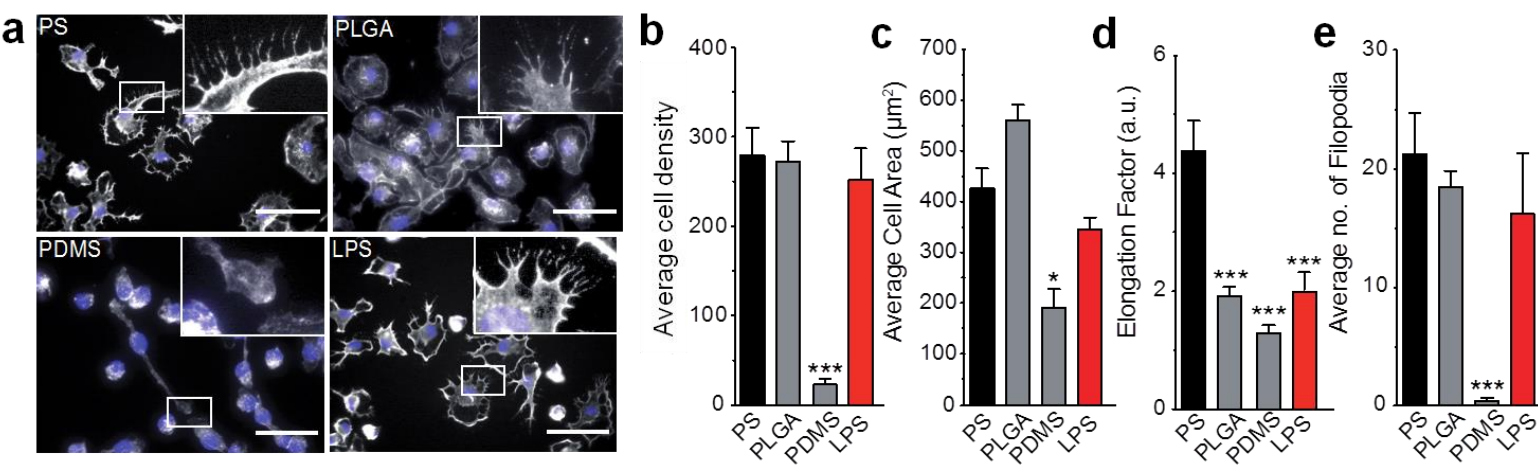

Figure 1. (a) Fluorescence images of MPs adhering to PS and polymer films in vitro 24 hours after plating. Insets evidence the development of filopodia. Cytoskeleton is labelled with Phalloidin (grey) and nuclei are labelled with DAPI (blue). Quantification of morphological features of MPs adhered to different substrates: (b) number of adherent cells, (c) average cell area, (d) elongation factor, (e) and number of filopodia. LPS-activated MPs were used as positive controls (red bars). 
a

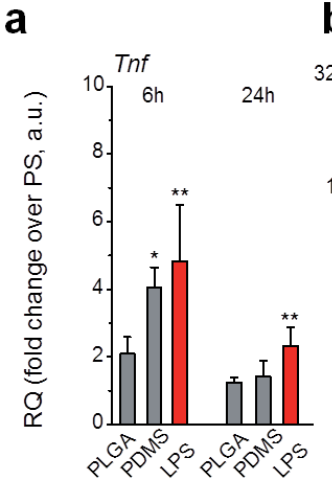

d

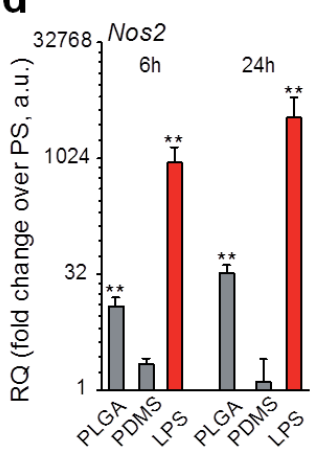

b
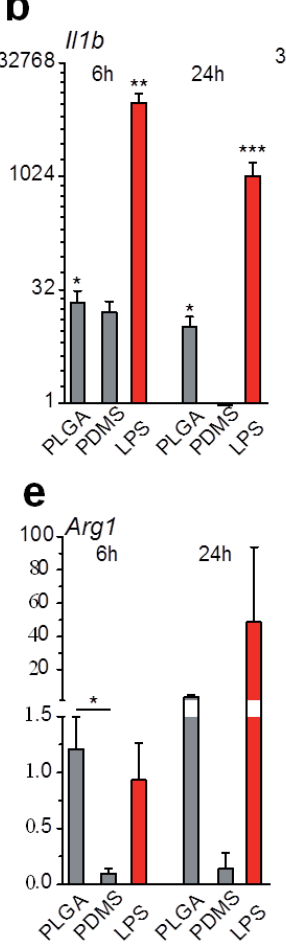

C

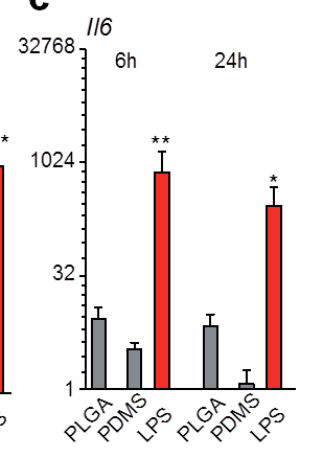

f

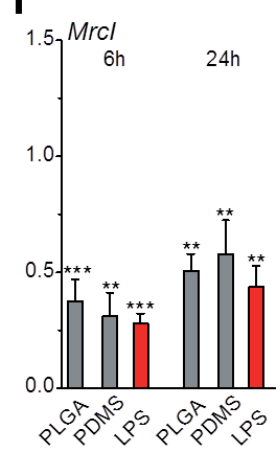

g

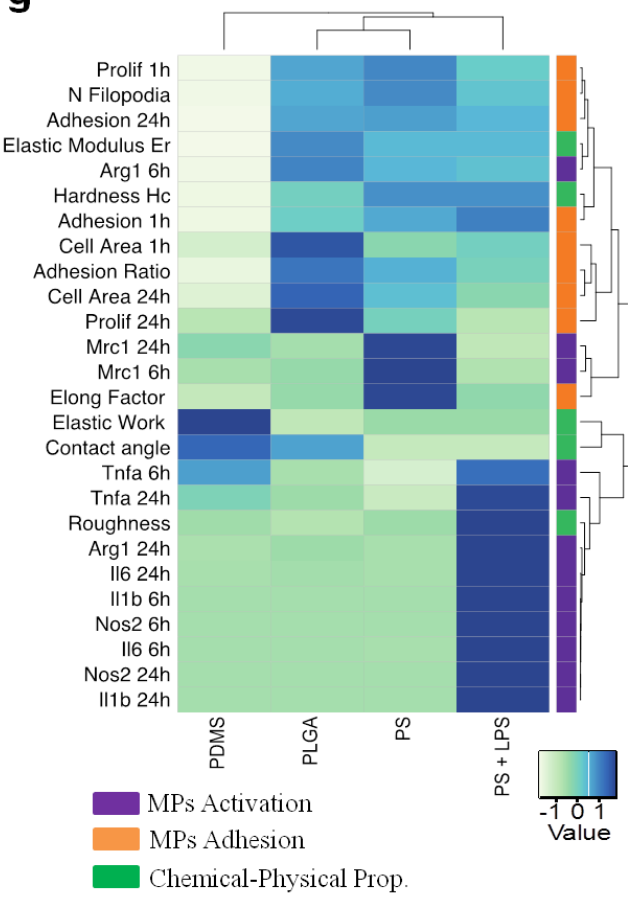

Figure 2 Analysis of inflammatory biomarkers expressed by MPs on PDMS and PLGA, and PS: (a-f) qRT-PCR analyses showing changes in the expression of $\operatorname{Tnf}(\mathbf{a}), \operatorname{Illb}(\mathbf{b}), \operatorname{Il6}$ (c) and Nos2 (d) (pro-inflammatory) and Argl (e) and MrcI (f) (anti-inflammatory) mRNAs in MPs at 6 and 24 hours in vitro. PS \pm LPS were positive and negative controls (black and red bars, respectively). Data were obtained from $n \geq 3$ independent experiments, and expressed as mean fold change (over PS) \pm SEM. Data were analysed with one-way-Anova and Bonferroni's post-test correction. LPS = lipopolysaccharide; RQ = Relative quantity. * $\mathrm{p} \leq 0.05$; ** $\mathrm{p} \leq 0.005 ; * * * \mathrm{p} \leq 0.001$, vs. PS. (g) Heatmap correlation of the immunogenic properties (as from Figure 2a-f) and chemical-physical properties of PDMS, PLGA, PS with and without LPS, as from Table 1. The color-scale represents rescaled values (as described in Methods). 


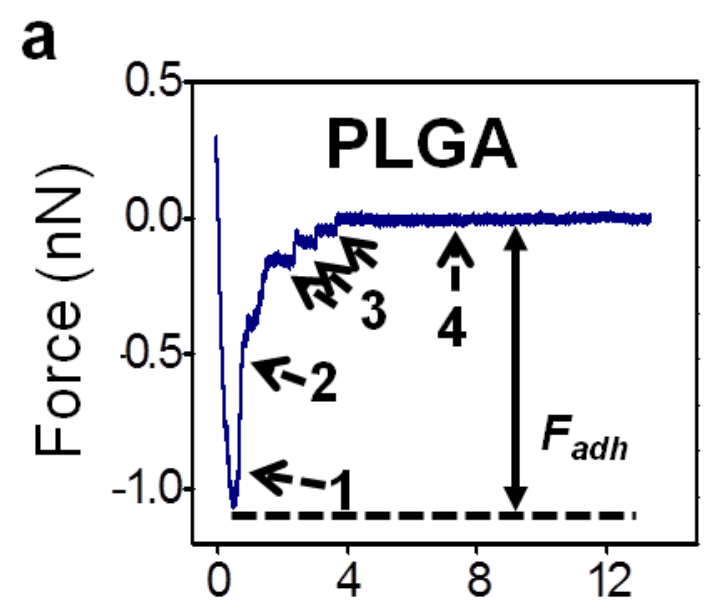

Piezo displacement $(\mu \mathrm{m})$ b

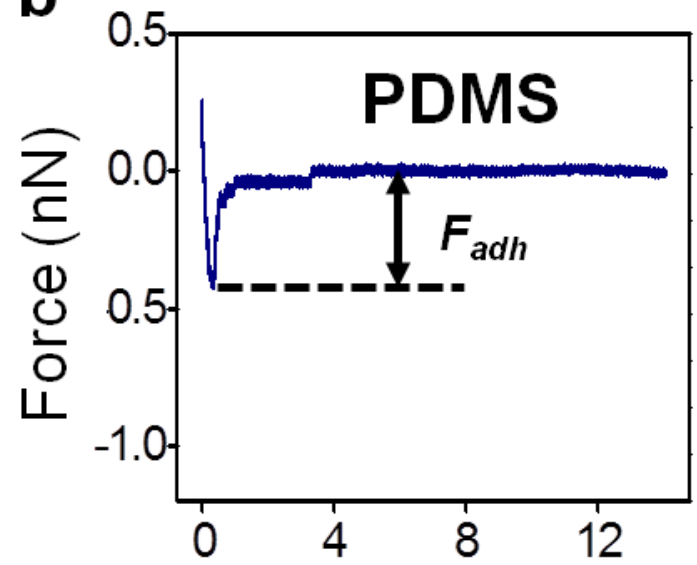

Piezo displacement $(\mu \mathrm{m})$

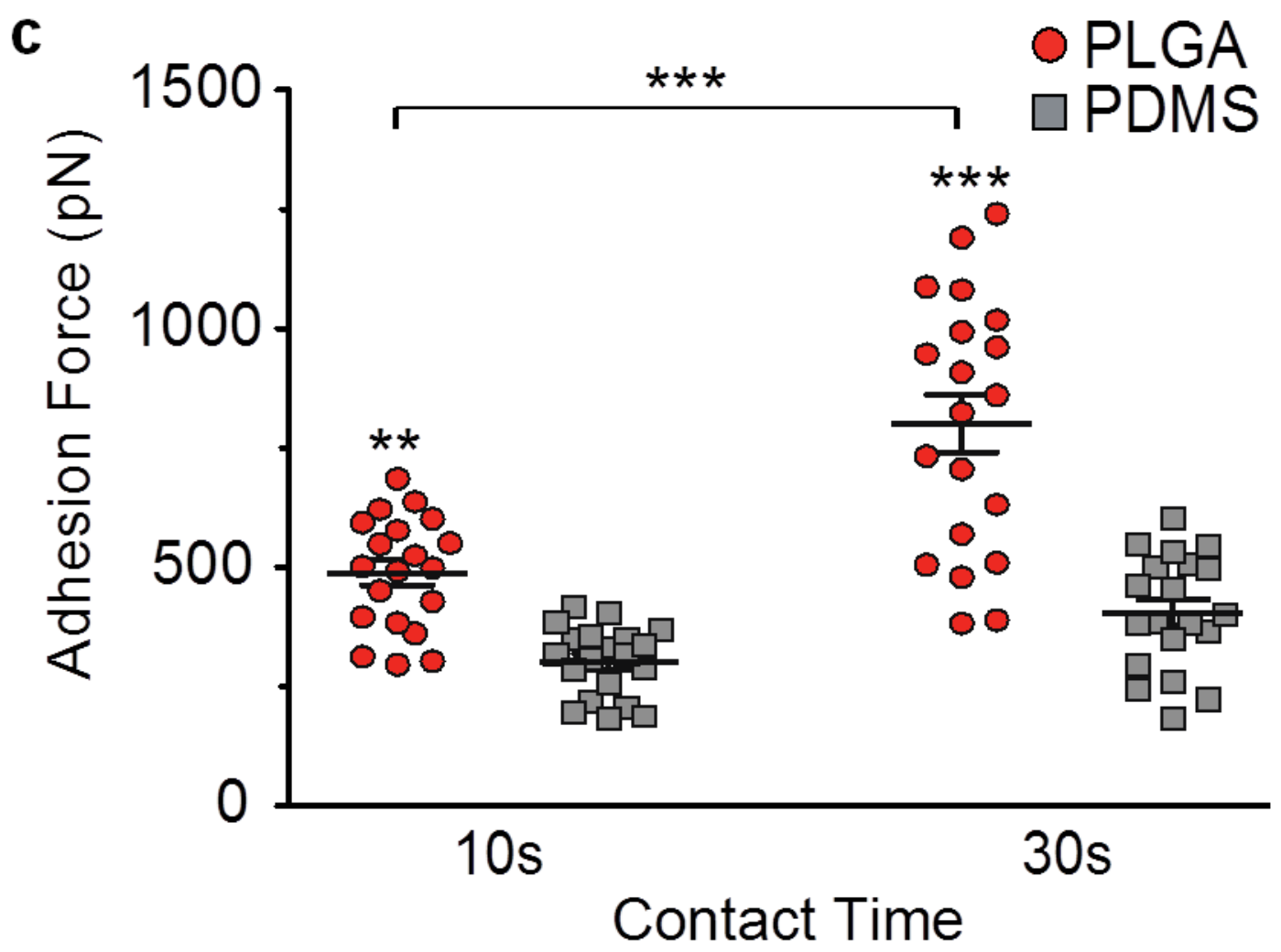

Figure 3 Typical force curve (retraction or unloading part) from representative SCFS experiments on PLGA (a) and PDMS (b). Key features are marked: (1) increase of the adhesion force corresponding to the elastic stretching of the entire cell; (2) de-adhesion steps, which can attributed to the unbinding of cell membrane proteins; (3) plateaus of constant force due to the detachment from the surface of micrometer-long membrane tethers; and (4) detachment of the cell from the polymer surface. (c) Quantification of adhesion force $(\mathrm{pN})$ on PLGA (red dots) and PDMS (grey squares) samples, at two different contact times (10 and 30 s). At least 15 cells were tested in alternating measurements for each material. Data are expressed as mean numbers \pm SEM and analyzed with one-way-Anova and Bonferroni's posttest correction. 


\section{WILEY-VCH}

a
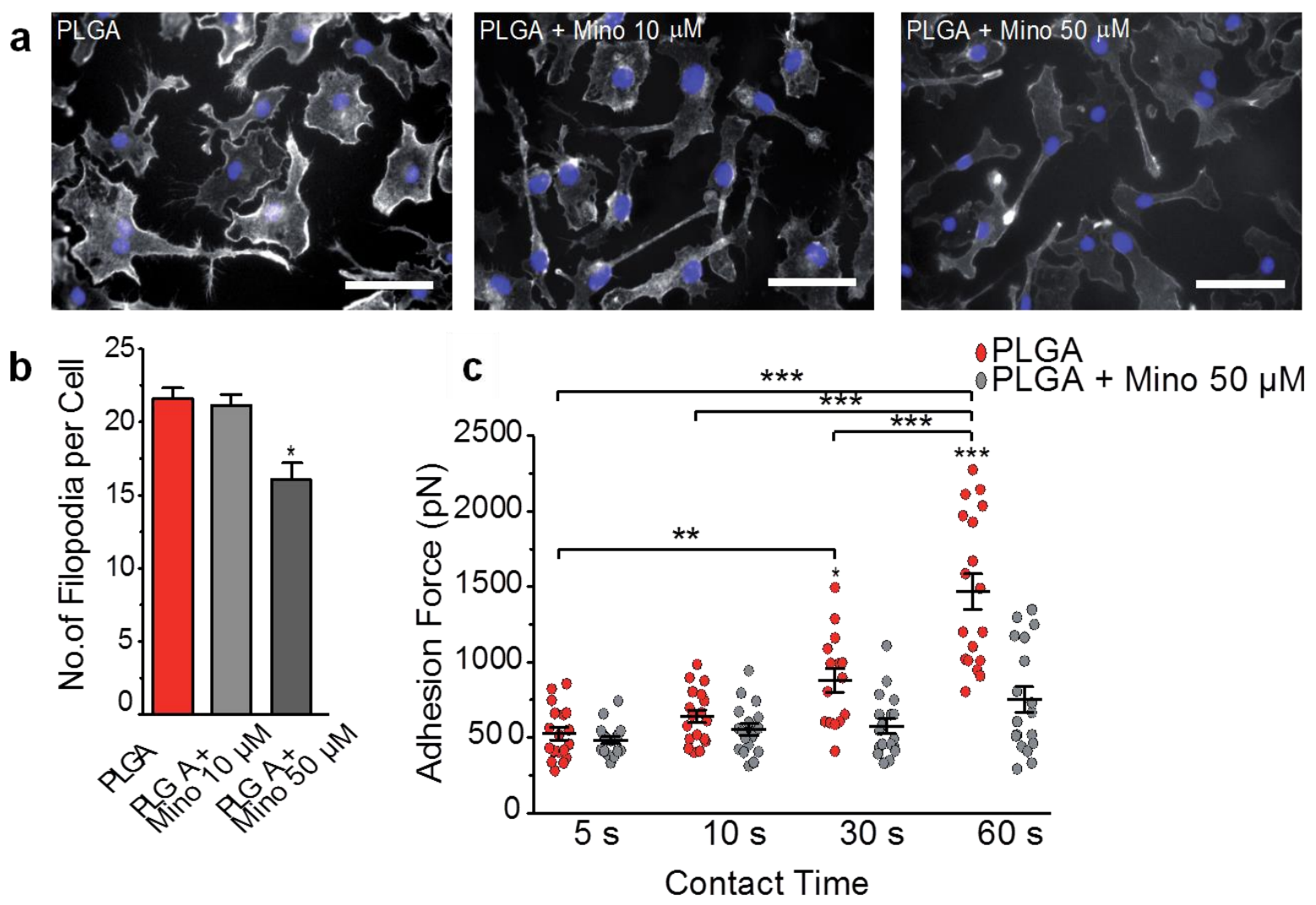

Figure 4. (a) Fluorescence images of MPs adhering to PLGA without Minocycline (left), 10 $\mu \mathrm{M}$ Minocycline (center), and $50 \mu \mathrm{M}$ Minocycline (right) after 24 hours in vitro. Cytoskeleton is labeled with Phalloidin (grey) and nuclei are labelled with DAPI (blue). (b) Quantification of filopodia formation 24 hours after plating on PLGA and PLGA + Minocycline as in A. (c) SCFS-based quantification of adhesion force to PLGA: control (red) vs. Minocycline-treated (grey) MPs at 5, 10, 30 and 60 seconds after contact. Scale bar in C: $50 \mu \mathrm{m} . * \mathrm{p} \leq 0.05 ; * * \mathrm{p} \leq 0.005 ; * * * \mathrm{p} \leq 0.001$, vs. controls 

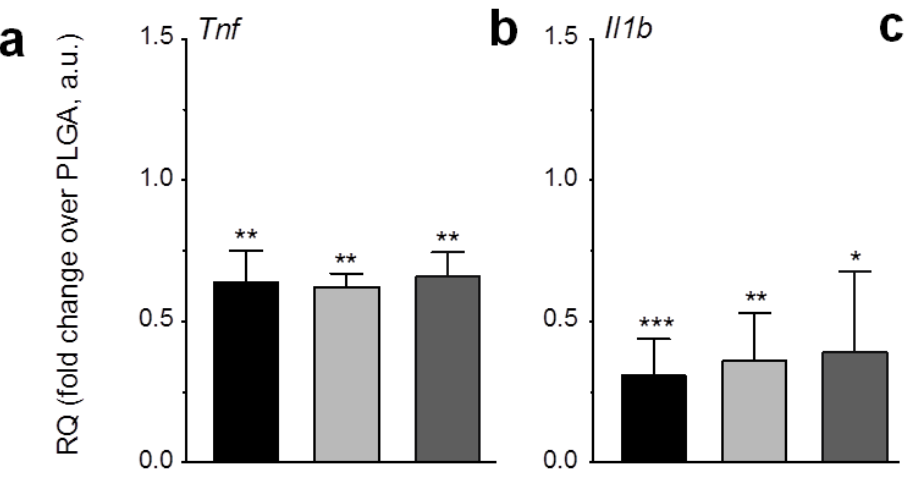

C

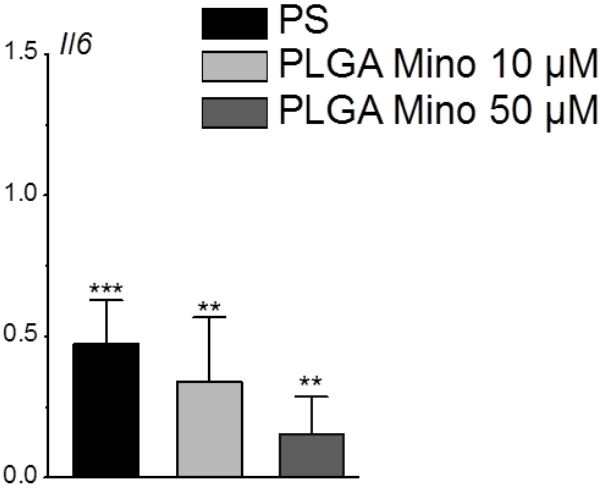

d
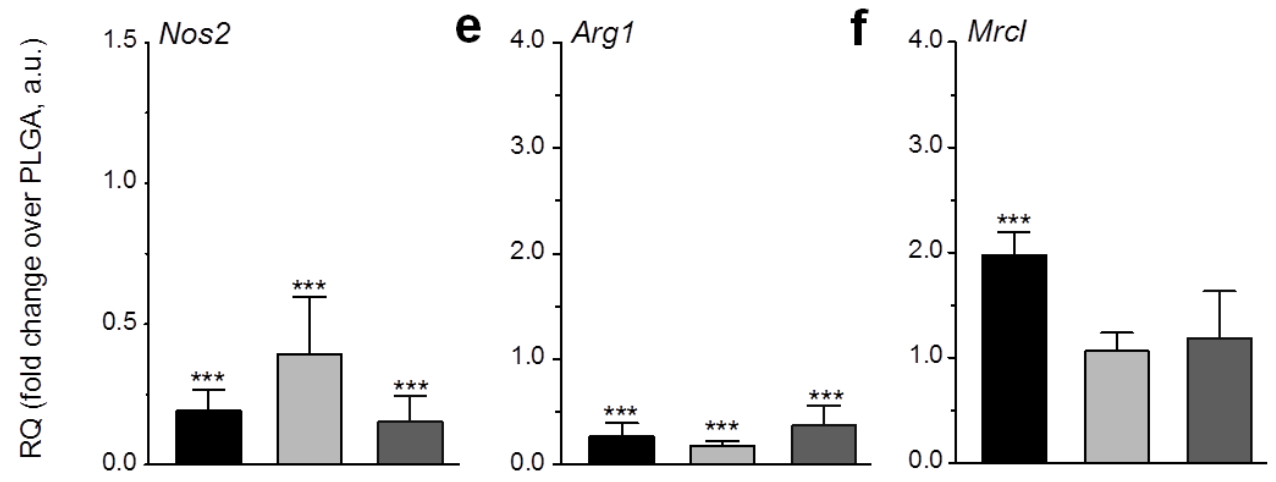

Figure 5. (a-f) qRT-PCR analyses showing changes in the expression of $\operatorname{Tnf}(\mathbf{a}), \operatorname{Illb}$ (b), Il6 (c),Nos2 (d) (pro-inflammatory) and Argl (e) and $\mathrm{MrcI}$ (f) (anti-inflammatory) mRNAs in MPs on PLGA after 24 hours with Minocycline 10 (light grey bars) or 50 (dark grey bars) $\mu \mathrm{M}$. MPs on PLGA only (black bars) were used as control. Mino = Minocycline; RQ $=$ Relative quantity. $* \mathrm{p} \leq 0.05 ; * * \mathrm{p} \leq 0.005 ; * * * \mathrm{p} \leq 0.001, v s$. PLGA. 
The table of contents entry should be 50-60 words long, and the first phrase should be bold.

Different polymers relevant for biomedical applications are screened for their ability to induce foreign body reaction (FBR) in vitro by a new quantitative local approach. Adhesion force by single-cell force spectroscopy (SCFS) is correlated to the expression of inflammatory biomarkers and morphology. The results also show that the anti-inflammatory drug Minocycline decreases the adhesion of macrophages on immunogenic poly-lactic-coglycolic acid (PLGA), preventing their activation to FBR.

Keywords: foreign body reaction, single cell force spectroscopy, mononuclear phagocytes, biomaterials, organic electronics, implants, Minocycline.

\section{Interfacing polymers and tissues: Quantitative local assessment of the Foreign Body \\ Reaction of Mononuclear Phagocytes to Polymeric Materials}

Elena Giusto ${ }^{a}$, Matteo Donegà ${ }^{a}$, Andra C. Dumitru ${ }^{b}$, Giulia Foschi ${ }^{c,+}$, Stefano Casalini ${ }^{c}$, Michele Bianchi ${ }^{d}$, Tommaso Leonardi a,e, Alessandro Russo ${ }^{d}$, Luigi G. Occhipintif ${ }^{f}$, Fabio Biscarini $^{c *}$, Ricardo Garcia ${ }^{b, *}$ and Stefano Pluchino, ${ }^{a, *}$
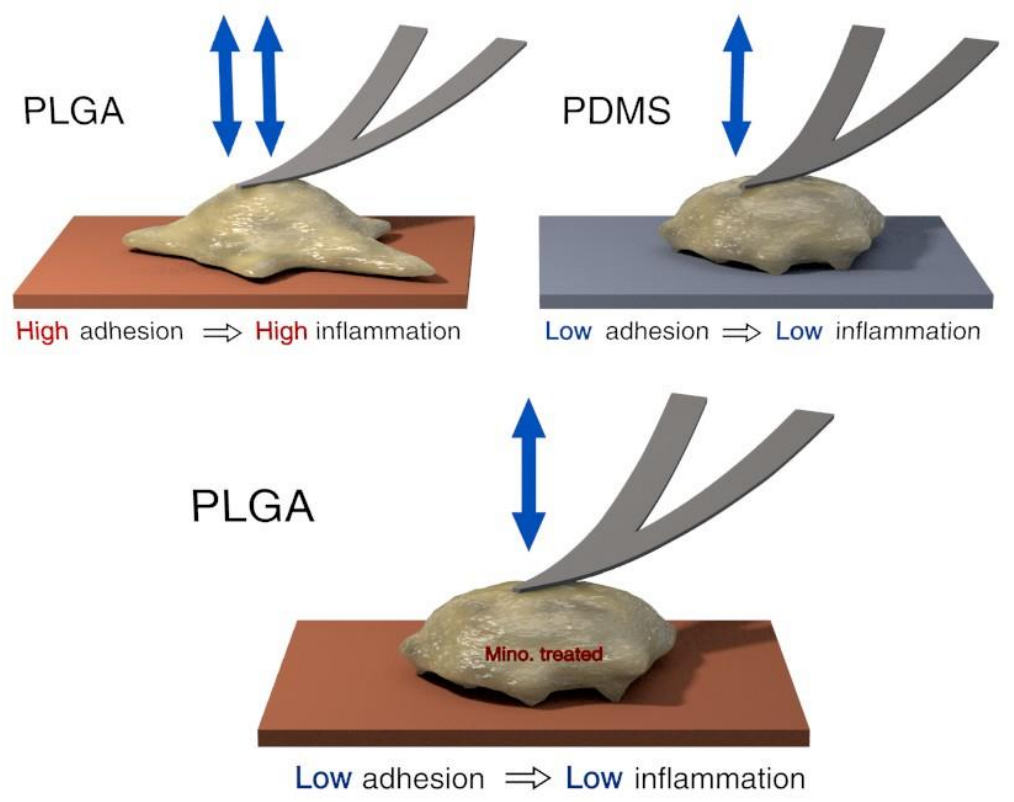

ToC figure 